\title{
Collateral damage of using colistin in hospitalized patients on emergence of colistin-resistant Escherichia coli and Klebsiella pneumoniae colonization and infection
}

\author{
W. Wangchinda ${ }^{1}$, N. Pati ${ }^{1}$, N. Maknakhon ${ }^{1}$, C. Seenama' ${ }^{1}$ S. Tiengrim² ${ }^{2}$ and V. Thamlikitkul ${ }^{1 *}$
}

\begin{abstract}
Background: Colistin has been used for therapy of carbapenem-resistant Gram-negative infections in Thailand, especially carbapenem-resistant A. baumannii and P. aeruginosa, for more than 10 years. However, the prevalence of colistin-resistant A. baumannii or P. aeruginosa is still less than $5 \%$. Colistin-resistant Enterobacteriaceae has been increasingly reported globally over the past few years and the use of colistin in food animals might be associated with an emergence of colistin resistance in Enterobacteriaceae. This study aimed to determine the effect of colistin exposure in hospitalized patients who received colistin on development of colistin-resistant (CoR) Escherichia coli (EC) or Klebsiella pneumoniae (KP) colonization and infection.

Methods: A prospective observational study was performed in adult hospitalized patients at Siriraj Hospital who received colistin for treatment of infections during December 2016 and November 2017. The surveillance culture samples were collected from the stool and the site of infection of each patient who received colistin at the study enrollment, days 3 and 7 after the study enrollment, and once a week thereafter for determination of CoR EC and CoR KP. CoR EC and CoR KP were also tested for a presence of mcr-1 gene.

Results: One hundred thirty-nine patients were included. Overall prevalence of COR EC or CoR KP colonization was 47.5\% among 139 subjects. Prevalence of CoR EC or CoR KP colonization was 17.3\% of subjects at study enrollment, and $30.2 \%$ after study enrollment. Use of fluoroquinolones, aminoglycosides, and colistin was found to be significantly associated with CoR EC or CoR KP colonization. The mcr-1 gene was detected in 13.0\% of CoR EC or CoR KP isolates, and in $27.3 \%$ of subjects with CoR EC or CoR KP colonization. CoR EC or CoR KP colonization persisted in $65.2 \%$ of the subjects at the end of the study. Five patients with CoR KP infections received combination antibiotics and they were alive at hospital discharge.

Conclusions: Prevalence of COR EC or COR KP colonization in hospitalized patients receiving colistin was high and it was associated with the use of colistin. Therefore, patients who receive colistin are at risk of developing CoR EC or CoR KP colonization and infection.
\end{abstract}

Keywords: Thailand, Prevalence, Colonization, Colistin-resistant Escherichia coli, Colistin-resistant Klebsiella pneumoniae, Colistin, Hospitalization

\footnotetext{
* Correspondence: visanu.tha@mahidol.ac.th

'Division of Infectious Diseases and Tropical Medicine, Department of

Medicine, Faculty of Medicine Siriraj Hospital, Mahidol University, 2 Wanglang

Road, Bangkoknoi, Bangkok 10700, Thailand
}

(c) The Author(s). 2018 Open Access This article is distributed under the terms of the Creative Commons Attribution 4.0 International License (http://creativecommons.org/licenses/by/4.0/), which permits unrestricted use, distribution, and reproduction in any medium, provided you give appropriate credit to the original author(s) and the source, provide a link to the Creative Commons license, and indicate if changes were made. The Creative Commons Public Domain Dedication waiver (http://creativecommons.org/publicdomain/zero/1.0/) applies to the data made available in this article, unless otherwise stated. 


\section{Background}

Colistin is one of the last-resort antibiotics available for treatment of infections caused by multidrug-resistant Gram-negative bacteria, including carbapenem-resistant Acinetobacter baumannii, carbapenem-resistant Pseudomonas aeruginosa, and carbapenem-resistant Enterobacteriaceae (CRE) [1]. Colistin is currently in wide use as a result of the increasing prevalence of infections caused by these bacteria. Moreover, colistin is widely used in livestock for prevention, control, and treatment of infections [2]. The prior use of colistin in humans and animals is associated with colistin resistance and/or increased colistin minimal inhibitory concentration (MIC) in Gram-negative bacteria [3-5]. A main mechanism of resistance to colistin in Gram-negative bacteria is mediated by modification of lipid A, which results in a reduction in colistin affinity for Gram-negative bacteria. Colistin resistance mechanisms are usually mediated by chromosome-encoded mutations in the PmrAB and PhoPQ two-component regulatory systems, or by MgrB inactivation [6]. The emergence of a plasmid-mediated gene (mcr-1) that encodes a protein conferring resistance to colistin in Enterobacteriaceae isolated from foods, animals, and patients was reported from China in 2015 [7]. Subsequently, mcr-1-producing colistin-resistant Enterobacteriaceae have been reported from many countries around the World [8]. Colistin use in food animals is suspected as the major driver of the emergence of this plasmid-mediated colistin-resistant gene [7]. Association between colistin use for therapy of carbapenem-resistant $A$. baumannii and $P$. aeruginosa infections in humans and the emergence of colistin-resistance in these bacteria has been reported [9-13]. However, the prevalence of and association between colistin use for therapy of carbapenem-resistant Gram-negative bacterial infections in humans and the emergence of colistin resistance in Enterobacteriaceae in individuals who receive colistin are not well-understood.

Accordingly, the aim of this study was to determine the effect of colistin use in hospitalized patients on emergence of colistin-resistant (CoR) E. coli (EC) and $K$. pneumoniae (KP) colonization and infection.

\section{Methods}

This prospective observational study was conducted at Siriraj Hospital, a 2300-bed university-based tertiary care hospital in Bangkok, Thailand, during December 2016 to November 2017. The study protocol was approved by the Siriraj Institutional Review Board, and written informed consent was obtained from all subjects.

Adult patients hospitalized in the medical wards or medical intensive care units at Siriraj Hospital who received colistin were included in this study. The decision to prescribe colistin treatment was made by each patient's attending physician. Each enrolled subject had a first surveillance stool or rectal swab culture sample collected as soon as possible within several days after receiving colistin. Subsequent stool or rectal swab samples were collected on days 3 and 7 after colistin treatment, and once a week thereafter until the patient left the hospital, died, or up to 4 weeks after receiving colistin if the previously collected samples did not reveal colistin-resistant (CoR) E. coli (EC) or K. pneumoniae (KP). Surveillance culture samples from the site of infection that precipitated the use of colistin therapy were also periodically collected if they were available. Collected surveillance culture samples were grown on a MacConkey agar plate. The lactose-fermenter bacterial colonies grown on primary MacConkey agar plate after overnight incubation at $35{ }^{\circ} \mathrm{C}$ with different morphology were subcultured. Identification of the targeted bacteria (EC and KP) was made by using traditional biochemical tests including growth on MacConkey agar, Kovac's oxidase, triple sugar iron agar reaction, indole production, methyl red, Voges-Proskauer, citrate utilization, production of hydrogen sulfide, urea hydrolysis, phenylalanine deaminase, lysine decarboxylase, ornithine decarboxylase, motility, malonate utilization, gas from glucose fermentation, lactose fermentation, manitol fermentation [14]. The minimum inhibitory concentration (MIC) of colistin was performed by broth microdilution method, and a colistin $\mathrm{MIC} \geq 4 \mathrm{mg} / \mathrm{L}$ was considered colistin resistance. The susceptibility of isolated CoR EC and CoR KP to ceftriaxone and ertapenem was also assessed by disk diffusion method. Determination of presence of the mcr-1 gene was performed in CoR EC and CoR KP isolates using polymerase chain reaction (PCR) amplification [7]. Screening for the mcr-1 gene was performed by PCR with forward (5'-GTGTGGTACCGACGCTCGG-3') and reverse (5'-CAAGCCCAATCGGCGCATC-3') primers. PCR amplification was performed using i-Taq DNA polymerase under the following cycling conditions: 34 cycles of $94{ }^{\circ} \mathrm{C}$ for $20 \mathrm{~s}, 50{ }^{\circ} \mathrm{C}$ for $20 \mathrm{~s}$, and $72{ }^{\circ} \mathrm{C}$ for $30 \mathrm{~s}$, followed by 1 cycle of $72{ }^{\circ} \mathrm{C}$ for $5 \mathrm{~min}$. PCR products were separated on a $1 \%$ agarose gel. The PCR amplicon size was approximately $460 \mathrm{bp}$.

Data collected from each subject included demographics, underlying diseases and conditions, type of isolated bacteria (true pathogen or colonizer), type of infection, previous antimicrobial treatment received during the 3-month period prior to study enrollment, antimicrobial treatment during the study period, and the outcome of colistin treatment. The dose, frequency and duration of colistin were also recorded.

The primary outcomes of this study were prevalence of colonization with CoR EC or CoR KP in subjects who received colistin during the study period, and the factors associated with CoR EC or CoR KP colonization. Colonization of CoR EC or CoR KP was defined as detection of CoR EC or CoR KP in surveillance culture samples in the absence of clinical features of infection at 
the site of the sample collection. CoR EC or CoR KP infection was defined according to the diagnosis of the patient's attending physician. The secondary outcomes were the clinical courses of colonization with CoR EC or CoR KP in patients who received colistin during the study period, the prevalence of the mcr-1 gene in isolated CoR EC and CoR KP, and the clinical courses of subjects with CoR EC or CoR KP colonization and infection.

\section{Sample size calculation and data analysis}

An overall colonization and infection rate of colistin-resistant Gram-negative bacteria in a cohort of critically-ill patients in intensive care units was 27\% [3]. Therefore, it was estimated that at least $30 \%$ of subjects who received colistin would develop colonization with CoR $\mathrm{EC}$ or CoR KP during the study period. Using a variation in prevalence of developing CoR EC or CoR KP colonization during the study period of $8 \%$ and an acceptable type 1 two-sided error of 5\%, a minimum sample size of 127 subjects was needed.

All data analyses were performed using SPSS Statistics version 16.0 (SPSS, Inc., Chicago, IL, USA). Demographics and clinical characteristics of patients were summarized using descriptive statistics. Categorical data are presented as frequency and percentage, and continuous data are shown as mean \pm standard deviation or median and range. Comparison of categorical data was performed using chi-square test or Fisher's exact test, with Student's t-test used to compare continuous data. A $p$-value less than or equal to 0.05 was considered statistically significant.

\section{Results}

\section{Study population}

A total of 139 subjects were included. All enrolled patients received colistin, all had $\geq 2$ stool or rectal swab samples collected, and all had their first sample collected within 5 days after the start of colistin treatment. The demographics and clinical characteristics of subjects are summarized in Table 1.

The mean age of subjects was 67.2 years, and the distribution between genders was not different. Most subjects had chronic underlying diseases or healthcare-associated conditions. Previous hospitalization within 3 months was observed in $52.5 \%$ of subjects. Lower respiratory tract infection was the most common site of infection, followed by blood stream infection and urinary tract infection. Colistin therapy was given to $79.1 \%$ of subjects with documented bacterial infections, and in $77.1 \%$ of episodes of documented bacterial infection. A. baumannii was the most common cause of documented infection, followed by $K$. pneumoniae and P. aeruginosa. E. coli and Enterobacter cloacae were found in only $4 \%$ of subjects. Most subjects received mechanical ventilation (84.2\%) or central venous catheterization (61.2\%). The mean APACHE II score was 21.8. Most patients received various antibiotics within 3 months prior to study enrollment, including carbapenems, cephalosporins, beta-lactam/beta-lactamase inhibitors, fluoroquinolones, vancomycin, aminoglycosides and colistin. The subjects received 166 courses of colistin during the study period. Colistin was given to 110 subjects (79.1\%) with documented infections, with 29 patients (20.9\%) being prescribed colistin as empirical therapy. The most frequent indication for colistin use was A. baumannii ventilator-associated pneumonia. The median duration of all colistin treatment courses was 9 days (range: 1-55).

\section{Colistin-resistant E. coli or K. pneumoniae}

The surveillance culture samples collected included 673 stool or rectal swab samples, 465 sputum samples, 103 urine samples, 2 percutaneous drainage samples, and 1 wound swab sample. Colistin resistance was observed in 246 isolates of EC and KP, of which 26 were EC and 220 were KP. One hundred and sixty-six isolates (67.5\%) of CoR EC or CoR KP were recovered from stool or rectal swab samples, while 70 isolates (28.5\%) and 10 isolates (4.1\%) were recovered from sputum samples and urine samples, respectively. The $\mathrm{MIC}_{50}, \mathrm{MIC}_{90}$, and $\mathrm{MIC}$ range of all CoR EC and CoR KP isolates was 64, 128, and 4 to $>128 \mathrm{mg} / \mathrm{L}$, respectively. The $\mathrm{MIC}_{50}, \mathrm{MIC}_{90}$, and $\mathrm{MIC}$ range of all CoR EC isolates was 8,64 , and 4 to $128 \mathrm{mg} / \mathrm{L}$, respectively. The $\mathrm{MIC}_{50}, \mathrm{MIC}_{90}$, and $\mathrm{MIC}$ range of all CoR KP isolates was 64,128 , and 4 to $>128 \mathrm{mg} / \mathrm{L}$, respectively. Among the 26 CoR EC isolates, $80.8 \%$ were resistant to ceftriaxone, and $11.5 \%$ were resistant to carbapenems. Ceftriaxone resistance and carbapenems resistance was observed in 96.8 and $75.5 \%$ of 220 CoR KP isolates, respectively.

\section{Colistin-resistant $E$. coli or $K$. pneumoniae colonization in all collected clinical samples}

The number of subjects colonized with CoR EC or CoR $\mathrm{KP}$ in the first surveillance culture samples at study enrollment, and the number of patients colonized with CoR EC or CoR KP in subsequent surveillance culture samples during the study period are shown in Fig. 1.

CoR EC or CoR KP colonization was detected in 66 of 139 subjects (47.5\%). Sixty of those patients (43.2\%) were colonized with CoR KP, and 13 subjects (9.4\%) were colonized with CoR EC. CoR EC or CoR KP colonization was detected in 24 subjects (17.3\%) at study enrollment, with 22 subjects $(15.8 \%)$ colonized by CoR KP and 2 subjects (1.4\%) colonized by CoR EC. Of the 115 subjects with no colonization of CoR EC or CoR KP at study enrollment, 42 subjects $(36.5 \%)$ subsequently developed colonization with CoR EC or CoR KP. Of those, 
Table 1 Demographics and clinical characteristics of 139 subjects

\begin{tabular}{|c|c|}
\hline Variables & Values \\
\hline Female gender, $\mathrm{n}(\%)$ & $70(50.4 \%)$ \\
\hline Age (yrs), mean $\pm S D$ & $67.2 \pm 15.9$ \\
\hline Comorbid conditions, n (\%) & $131(94.2 \%)$ \\
\hline Diabetes mellitus & $53(38.1 \%)$ \\
\hline Cardiovascular diseases & $41(29.5 \%)$ \\
\hline Renal diseases & $37(26.6 \%)$ \\
\hline Cerebrovascular diseases & 35 (25.2\%) \\
\hline Solid malignancy & $21(15.1 \%)$ \\
\hline Chronic pulmonary diseases & $20(14.3 \%)$ \\
\hline Hematologic malignancy & $16(11.5 \%)$ \\
\hline Liver diseases & $10(7.2 \%)$ \\
\hline Organ transplantation & $4(2.9 \%)$ \\
\hline HIV infection & $2(1.4 \%)$ \\
\hline $\begin{array}{l}\text { Previous hospitalization within } \\
3 \text { months, } \mathrm{n}(\%)\end{array}$ & $73(52.5 \%)$ \\
\hline \multicolumn{2}{|l|}{$\begin{array}{l}\text { Site of infection in } 139 \text { patients } \\
\text { at enrollment, } \mathrm{n}(\%)\end{array}$} \\
\hline Lower respiratory tract infection & $93(66.9 \%)$ \\
\hline Blood stream infection & $40(28.8 \%)$ \\
\hline Urinary tract infection & $18(12.9 \%)$ \\
\hline Gastrointestinal tract infection & $11(7.9 \%)$ \\
\hline Skin and soft tissue infection & $8(5.8 \%)$ \\
\hline Surgical site infection & $1(0.7 \%)$ \\
\hline Bone and joint infection & $1(0.7 \%)$ \\
\hline
\end{tabular}

Documented bacteria-caused infections in 110 patients that required colistin therapy, n (\%)

$$
\begin{aligned}
& \text { A. baumannii } \\
& \text { K. pneumoniae } \\
& \text { P. aeruginosa } \\
& \text { E. coli } \\
& \text { E. cloacae }
\end{aligned}
$$

Medical procedure, n (\%)

Mechanical ventilation

$117(84.2 \%)$

Central venous catheterization

$85(61.2 \%)$

Chronic intermittent hemodialysis

$53(38.1 \%)$

Severity of patient illness, mean \pm SD

APACHE II score at enrollment

$21.8 \pm 7.4$

Antibiotic use for the current hospitalization prior to study enrollment, $\mathrm{n}(\%)$

Carbapenems
Cephalosporins
Beta-lactam/beta-lactamase inhibitors
Fluoroquinolones

Table 1 Demographics and clinical characteristics of 139 subjects (Continued)

\begin{tabular}{ll}
\hline Variables & Values \\
\hline Vancomycin & $43(30.9 \%)$ \\
Aminoglycosides & $6(4.3 \%)$ \\
Colistin & $9(6.8 \%)$ \\
Colistin use within 3 months prior to & $15(10.8 \%)$ \\
study enrollment, n (\%) & \\
\hline
\end{tabular}

39 subjects $(33.9 \%)$ were colonized with CoR KP, and 8 (7.0\%) were colonized with CoR EC.

\section{Comparison of subjects with and without colistin- resistant $E$. coli or K. pneumoniae colonization during the study period}

Comparison of subjects with (66 patients) and without (73 patients) CoR EC or CoR KP colonization during the study period is shown in Table 2 .

The duration from hospital admission to the first surveillance culture sample collection, and the duration from study enrollment to the first surveillance culture sample collection during the study period were not significantly different between groups. The use of fluoroquinolones, aminoglycosides, or colistin within 3 months prior to study enrollment, and the amount, duration, and courses of colistin were all significantly greater in subjects with CoR EC or CoR KP colonization than in those without CoR EC or CoR KP colonization (all $p<0.05$ ). The median and range of duration of the presence of CoR EC or CoR KP colonization was 13 days and 1-68 days, respectively. Neither CoR EC nor CoR KP was isolated from 23 subjects (34.8\%) at the end of the study period, whereas 43 patients $(65.2 \%)$ had persistent CoR EC or CoR KP colonization at the end of the study period. The median and range of duration of colistin use in subjects that had no CoR EC or CoR KP colonization at the end of the study was 11 days and 2-57 days, respectively. The corresponding values for patients who had persistent CoR EC or CoR KP colonization at the end of the study were 14 days and $3-65$ days, respectively $(p=0.43)$. The length of hospital stay in subjects with CoR EC or CoR KP colonization was significantly longer than in subjects without CoR EC or CoR KP colonization. The rates of all-cause mortality at hospital discharge were similar between subjects with and without CoR EC or CoR KP colonization.

\section{Comparison of subjects with and without colistin- resistant $E$. coli or K. pneumoniae colonization at study enrollment}

CoR EC or CoR KP colonization was detected in 24 subjects $(17.3 \%)$ at study enrollment. Twenty-two subjects (15.8\%) were colonized with CoR KP, and 2 patients (1.4\%) were colonized with CoR EC. Comparison of 


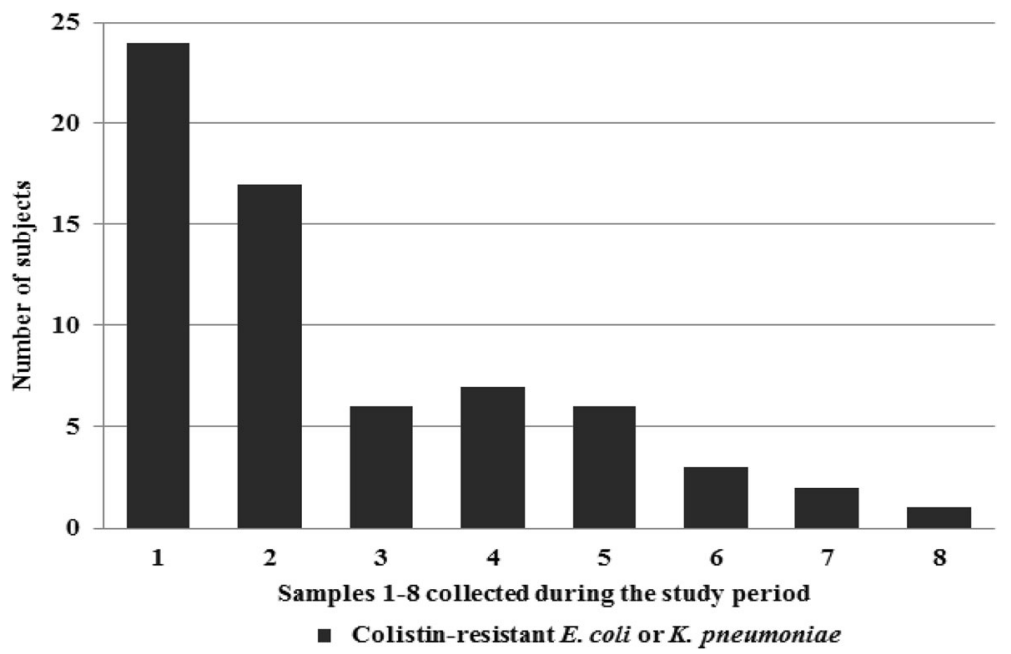

Fig. 1 Number of subjects colonized with colistin-resistant E. coli or K. pneumoniae in surveillance culture samples at each of 8 sample collections during the study period

subjects with and without CoR EC or CoR KP colonization at study enrollment is shown in Table 3.

Duration from hospital admission to study enrollment, and duration from study enrollment to first surveillance culture sample collection between subjects with and without CoR EC or CoR KP colonization were not significantly different between groups. The use of fluoroquinolones or vancomycin for the current hospitalization prior to study enrollment and surgery were significantly more prevalent in subjects with CoR EC or CoR KP colonization at study enrollment than in those without CoR EC or CoR KP colonization at study enrollment. The use of aminoglycosides for the current hospitalization prior to study enrollment and colistin use within 3 months prior to study enrollment were observed more often in subjects with CoR EC or CoR KP colonization at study enrollment. The median and range of the duration of the presence of CoR EC or CoR KP colonization were 18.5 days and 2-68 days, respectively. CoR EC or CoR KP was not isolated from $25.0 \%$ of 24 subjects at the end of the study period. In contrast, the remaining $75.0 \%$ of those subjects had persistent CoR EC or CoR KP colonization at the end of the study period. Length of hospital stay and the rate of all-cause mortality at hospital discharge in subjects with CoR EC or CoR KP colonization at study enrollment were both greater than in patients without CoR EC or CoR KP colonization at study enrollment.

Comparison of subjects with and without colistin-resistant E. coli or K. pneumoniae colonization after study enrollment among subjects without colonization by colistin-resistant $E$. coli or K. pneumoniae at study enrollment

Of the 115 subjects without colonization of CoR EC or CoR $\mathrm{KP}$ at study enrollment, 42 patients (36.5\%) subsequently developed colonization with CoR EC or CoR KP.
Comparison of subjects with and without CoR EC or CoR KP colonization after study enrollment among subjects without colonization by CoR EC or CoR KP at study enrollment is shown in Table 4.

Duration from hospital admission to the first surveillance culture sample collection, and duration from study enrollment to the first surveillance culture sample collection in patients with and without CoR EC or CoR KP colonization after study enrollment were not significantly different between groups. The use of colistin within 3 months prior to study enrollment, and the amount, duration, and courses of colistin were all significantly greater in patients with CoR EC or CoR KP colonization after study enrollment than in those without CoR EC or CoR KP colonization after study enrollment. The median and range of the time from study enrollment to the first detection of CoR EC or CoR KP colonization were 10 days and 3-42 days, respectively, and the mean duration was 14 days for CoR KP and 14 days for CoR EC. The median and range of the duration of the presence of CoR EC or CoR KP colonization was 7 days and 157 days, respectively. At the end of the study, CoR EC or CoR KP was and was not isolated from 59.5 and $40.5 \%$ of 42 subjects, respectively. Length of hospital stay in subjects with CoR EC or CoR KP colonization after study enrollment was significantly longer than in subjects without CoR EC or CoR KP colonization after study enrollment. The rate of all-cause mortality at hospital discharge was similar between subjects with and without CoR EC or CoR KP colonization after study enrollment.

Presence of mcr-1 gene among colistin-resistant $E$. coli and $K$. pneumoniae isolates

The mcr-1 gene was detected in $13.0 \%$ of 246 isolates of CoR EC or CoR KP. It was detected in $57.7 \%$ of 26 CoR 
Table 2 Comparison of subjects with and without colistin-resistant E. coli or K. pneumoniae colonization during the study period

\begin{tabular}{|c|c|c|c|}
\hline Variables & $\begin{array}{l}\text { Colonization } \\
(n=66)\end{array}$ & $\begin{array}{l}\text { No Colonization } \\
(n=73)\end{array}$ & $p$-value \\
\hline Female gender (yrs), n (\%) & $33(50.0 \%)$ & $37(50.7 \%)$ & 0.94 \\
\hline Age (yrs), mean $\pm S D$ & $65.7 \pm 16.7$ & $68.5 \pm 15.1$ & 0.32 \\
\hline $\begin{array}{l}\text { Duration from hospital admission to first sample } \\
\text { collection (days), median (range) }\end{array}$ & $16(1-106)$ & $16(2-81)$ & 0.37 \\
\hline $\begin{array}{l}\text { Duration from enrollment to first sample collection } \\
\text { (days), median (range) }\end{array}$ & $1(1-4)$ & $1(1-5)$ & 0.54 \\
\hline ICU admission, n (\%) & $24(36.4 \%)$ & $24(32.9 \%)$ & 0.67 \\
\hline Previous hospitalization, n (\%) & $36(54.5 \%)$ & $37(50.7 \%)$ & 0.65 \\
\hline APACHE II score at study enrollment, mean \pm SD & $21.6 \pm 7.1$ & $21.9 \pm 7.6$ & 0.77 \\
\hline Mechanical ventilation, n (\%) & $59(89.4 \%)$ & $58(79.5 \%)$ & 0.11 \\
\hline Indwelling central venous catheter, $\mathrm{n}(\%)$ & $45(68.2 \%)$ & $40(54.8 \%)$ & 0.11 \\
\hline Surgery, n (\%) & $45(68.2 \%)$ & 39 (53.4\%) & 0.08 \\
\hline Chronic intermittent hemodialysis, n (\%) & $8(12.1 \%)$ & $10(13.7 \%)$ & 0.78 \\
\hline \multicolumn{4}{|l|}{$\begin{array}{l}\text { Antibiotic use for the current hospitalization prior to } \\
\text { study enrollment and during study period, } \mathrm{n}(\%)\end{array}$} \\
\hline Carbapenems & $57(86.4 \%)$ & $66(90.4 \%)$ & 0.46 \\
\hline Cephalosporins & $31(47.0 \%)$ & $31(42.5 \%)$ & 0.59 \\
\hline Beta-lactam/ beta-lactamase inhibitors & $54(81.8 \%)$ & $52(71.2 \%)$ & 0.14 \\
\hline Fluoroquinolones & $42(63.6 \%)$ & $33(45.2 \%)$ & 0.03 \\
\hline Vancomycin & $40(60.6 \%)$ & $38(52.1 \%)$ & 0.31 \\
\hline Aminoglycosides & $10(15.2 \%)$ & $2(2.7 \%)$ & $<0.001$ \\
\hline \multicolumn{4}{|l|}{$\begin{array}{l}\text { Antibiotic use for the current hospitalization prior to } \\
\text { study enrollment, } n(\%)\end{array}$} \\
\hline Carbapenems & $52(78.8 \%)$ & $60(82.2 \%)$ & 0.61 \\
\hline Cephalosporins & $26(39.4 \%)$ & $30(41.1 \%)$ & 0.84 \\
\hline Beta-lactam/beta-lactamase inhibitors & $37(56.1 \%)$ & $38(52.1 \%)$ & 0.64 \\
\hline Fluoroquinolones & $24(36.4 \%)$ & $15(20.5 \%)$ & 0.04 \\
\hline Vancomycin & $22(33.3 \%)$ & $21(28.8 \%)$ & 0.56 \\
\hline Aminoglycosides & $5(7.6 \%)$ & $1(1.4 \%)$ & 0.10 \\
\hline Colistin use within 3 months prior to study enrollment, n (\%) & $13(19.7 \%)$ & $2(2.7 \%)$ & 0.001 \\
\hline Total amount of colistin (mg), median (range) & $3550(400-12,550)$ & $2000(500-10,650)$ & $<0.001$ \\
\hline Total duration of colistin use (days), median (range) & $13.5(2-58)$ & $9(2-32)$ & $<0.001$ \\
\hline Total courses of colistin therapy, mean \pm SD & $1.4 \pm 0.8$ & $1.1 \pm 0.4$ & 0.003 \\
\hline Total courses of colistin therapy, range & $1-4$ & $1-3$ & \\
\hline Patients who received more than 1 course of colistin therapy, n (\%) & $19(28.8 \%)$ & $7(9.6 \%)$ & 0.004 \\
\hline Length of hospital stay (days), median (range) & $46.5(12-246)$ & $32(6-192)$ & 0.01 \\
\hline All-cause mortality at hospital discharge, $\mathrm{n}(\%)$ & $35(53.0 \%)$ & $35(47.9 \%)$ & 0.55 \\
\hline
\end{tabular}

EC isolates, while only $7.7 \%$ of $220 \mathrm{CoR} \mathrm{KP}$ isolates showed presence of mcr-1. The colistin MIC of CoR EC isolates with the mcr-1 gene ranged from 4 to $16 \mathrm{mg} / \mathrm{L}$ (median: $8 \mathrm{mg} / \mathrm{L}$ ), whereas the colistin MIC of CoR KP isolates with the mcr-1 gene ranged from 4 to $>128 \mathrm{mg} / \mathrm{L}$ (median: $32 \mathrm{mg} / \mathrm{L}$ ). All isolates of CoR EC or CoR KP with the mcr-1 gene were colonizers. Presence of the mcr-1 gene was observed in $27.3 \%$ of the 66 subjects that had
CoR EC or CoR KP. The mcr-1 gene was detected in $12.5 \%$ of 24 subjects with CoR EC or CoR KP at study enrollment, and in $35.7 \%$ of 42 subjects with colonization with CoR EC or CoR KP after study enrollment.

\section{Colistin-resistant $E$. coli or K. pneumoniae infection}

Five subjects had infections caused by CoR KP. Of those, three had infections at study enrollment, and 2 developed 
Table 3 Comparison of subjects with and without colistin-resistant E. coli or K. pneumoniae colonization at study enrollment

\begin{tabular}{|c|c|c|c|}
\hline Variables & $\begin{array}{l}\text { Colonization at enrollment } \\
(n=24)\end{array}$ & $\begin{array}{l}\text { No colonization at enrollment } \\
(n=115)\end{array}$ & $p$-value \\
\hline Female gender & $13(54.2 \%)$ & $57(49.6 \%)$ & 0.68 \\
\hline Age (yrs), mean $\pm S D$ & $64.3 \pm 18.8$ & $67.8 \pm 15.2$ & 0.33 \\
\hline ICU admission, n (\%) & $9(37.5 \%)$ & $39(33.9 \%)$ & 0.77 \\
\hline Previous hospitalization, n (\%) & $15(62.5 \%)$ & $58(50.4 \%)$ & 0.28 \\
\hline Duration from hospital admission to enrollment (days), median (range) & $19.5(4-106)$ & $15(1-86)$ & 0.10 \\
\hline Duration from enrollment to first sample collection (days), median (range) & $1.5(1-4)$ & $1(1-5)$ & 0.48 \\
\hline APACHE II score at study enrollment, mean \pm SD & $20.6 \pm 7.2$ & $21.9 \pm 7.4$ & 0.65 \\
\hline Mechanical ventilation, n (\%) & $20(83.3 \%)$ & $97(84.3 \%)$ & 1.0 \\
\hline Indwelling central venous catheter, n (\%) & $17(70.8 \%)$ & $68(59.1 \%)$ & 0.29 \\
\hline Surgery, n (\%) & $19(79.2 \%)$ & $65(56.5 \%)$ & 0.04 \\
\hline Chronic intermittent hemodialysis, n (\%) & $4(16.7 \%)$ & $14(12.2 \%)$ & 0.74 \\
\hline Colistin use within 3 months prior to study enrollment, n (\%) & $4(16.7 \%)$ & $11(9.6 \%)$ & 0.47 \\
\hline \multicolumn{4}{|l|}{ Antibiotic use for the current hospitalization prior to study enrollment, n (\%) } \\
\hline Carbapenems & $20(83.3 \%)$ & $92(80.0 \%)$ & 0.79 \\
\hline Cephalosporins & $9(37.5 \%)$ & $47(40.9 \%)$ & 0.76 \\
\hline Beta-lactam/beta-lactamase inhibitors & $16(66.7 \%)$ & $59(51.3 \%)$ & 0.17 \\
\hline Fluoroquinolones & $13(54.2 \%)$ & $26(22.6 \%)$ & 0.002 \\
\hline Vancomycin & $13(54.2 \%)$ & $30(26.1 \%)$ & 0.007 \\
\hline Aminoglycosides & $3(12.5 \%)$ & $3(2.6 \%)$ & 0.06 \\
\hline Colistin & $3(13.6 \%)$ & $6(5.5 \%)$ & 0.36 \\
\hline Length of hospital stay (days), median (range) & $49.5(13-138)$ & $36(6-246)$ & 0.17 \\
\hline All-cause mortality at hospital discharge, $\mathrm{n}(\%)$ & $16(66.7 \%)$ & $54(47.0 \%)$ & 0.08 \\
\hline
\end{tabular}

infections after the start of the study. Of the 3 subjects with infections caused by CoR KP at study enrollment, 2 had concurrent CoR KP colonization. Of the 2 subjects with infections caused by CoR KP after study enrollment, 1 had concurrent CoR KP colonization and the other had concurrent CoR EC colonization. The sites of infections were urinary tract infections (3 patients), ventilator-associated pneumonia (1 patient) and bacteremia (1 patient). All subjects received colistin in combination with either fosfomycin or aminoglycoside, and they were alive at hospital discharge. There was no infection caused by CoR EC among subjects who had CoR EC colonization throughout the study period.

\section{Discussion}

The overall CoR EC or CoR KP colonization rate of $47.5 \%$ among subjects who received colistin in the present study was relatively high, especially the development of CoR EC or CoR KP colonization after study enrollment when compared with data from previous reports [15-17]. However, the study populations in those previous reports were general hospitalized patients, and they did not limit their studies to only patients who received colistin. To the best of our knowledge, the present study is the first to examine the prevalence of CoR EC or CoR KP colonization among hospitalized patients who received colistin. The prevalence of CoR EC or CoR KP in surveillance culture samples collected from these subjects was also high. It should be noted that a much larger number of CoR KP isolates than CoR EC isolates was recovered from subjects in this study (220 isolates vs. 26 isolates, respectively).

Many factors may have contributed to the development of CoR EC and CoR KP colonization observed in this study. The subjects with CoR EC or CoR KP colonization at study enrollment may have acquired colistin-resistant bacteria from the community prior to hospitalization. The probability of community-acquired CoR EC or CoR KP is low, however, because it is extremely rare for individuals, foods, and the environment in the community in Thailand to harbor CoR EC or CoR KP $[18,19]$. CoR EC and CoR KP have been detected at food animal farms in Thailand (especially swine farms) that use colistin extensively in the feed given to the swine to prevent and treat infection (V. Thamlikitkul. unpublished data). Most of the colistin-resistant Enterobacteriaceae found at swine farms in Thailand was $E$. coli while the prevalence of CoR EC isolates observed in 
Table 4 Comparison of subjects with and without colistin-resistant E. coli or K. pneumoniae colonization after study enrollment among subjects without colonization with colistin-resistant $E$. coli or K. pneumoniae at study enrollment

\begin{tabular}{|c|c|c|c|}
\hline Variables & Colonization after enrollment $(n=42)$ & No Colonization after enrollment $(n=73)$ & $p$-value \\
\hline Female gender (yrs), n (\%) & $20(47.6 \%)$ & $37(50.7 \%)$ & 0.75 \\
\hline Age (yrs), mean $\pm S D$ & $66.6 \pm 15.6$ & $68.5 \pm 15.1$ & 0.54 \\
\hline ICU admission, n (\%) & $15(35.7 \%)$ & $24(32.9 \%)$ & 0.76 \\
\hline Previous hospitalization, n (\%) & $21(50.0 \%)$ & $37(50.7 \%)$ & 0.94 \\
\hline APACHE II score at study enrollment, mean \pm SD & $21.8 \pm 7.2$ & $21.9 \pm 7.6$ & 0.92 \\
\hline Mechanical ventilation, n (\%) & 39 (92.9\%) & $58(79.5 \%)$ & 0.06 \\
\hline Indwelling central venous catheter, n (\%) & $28(66.7 \%)$ & $40(54.8 \%)$ & 0.21 \\
\hline Surgery, n (\%) & $26(61.9 \%)$ & $39(53.4 \%)$ & 0.38 \\
\hline Chronic intermittent hemodialysis, n (\%) & $4(9.5 \%)$ & $10(13.7 \%)$ & 0.51 \\
\hline \multicolumn{4}{|c|}{ Antibiotic use for current hospitalization prior to study enrollment and during study period, $\mathrm{n}(\%)$} \\
\hline Carbapenems & $35(83.3 \%)$ & $66(90.4 \%)$ & 0.26 \\
\hline Cephalosporins & $20(47.6 \%)$ & $31(42.5 \%)$ & 0.59 \\
\hline Beta-lactam/beta-lactamase inhibitors & $33(78.6 \%)$ & $52(71.2 \%)$ & 0.39 \\
\hline Fluoroquinolones & $25(59.5 \%)$ & $33(45.2 \%)$ & 0.14 \\
\hline Vancomycin & $23(54.8 \%)$ & $38(52.1 \%)$ & 0.78 \\
\hline Aminoglycosides & $5(11.9 \%)$ & $2(2.7 \%)$ & 0.10 \\
\hline \multicolumn{4}{|c|}{ Antibiotic use for the current hospitalization after study enrollment, $\mathrm{n}(\%)$} \\
\hline Carbapenems & $19(45.2 \%)$ & $34(46.6 \%)$ & 0.89 \\
\hline Cephalosporins & $6(14.3 \%)$ & $4(5.5 \%)$ & 0.17 \\
\hline Beta-lactam/beta-lactamase inhibitors & $25(59.5 \%)$ & $36(49.3 \%)$ & 0.29 \\
\hline Fluoroquinolones & $19(45.2 \%)$ & $25(34.2 \%)$ & 0.24 \\
\hline Vancomycin & $17(40.5 \%)$ & $27(37.0 \%)$ & 0.71 \\
\hline Aminoglycosides & $4(9.5 \%)$ & $2(2.7 \%)$ & 0.19 \\
\hline $\begin{array}{l}\text { Colistin use within } 3 \text { months prior to study } \\
\text { enrollment, } \mathrm{n}(\%)\end{array}$ & $9(21.4 \%)$ & $2(2.7 \%)$ & 0.002 \\
\hline Total amount of colistin (mg), median (range) & $2800(400-9350)$ & $2000(300-10,650)$ & 0.03 \\
\hline Total duration of colistin use (days), median (range) & $13(2-55)$ & $9(1-32)$ & 0.03 \\
\hline Total courses of colistin therapy, mean \pm SD & $1.3 \pm 0.6$ & $1.1 \pm 0.34$ & 0.02 \\
\hline Total courses of colistin therapy, range & $1-3$ & $1-3$ & \\
\hline $\begin{array}{l}\text { Patients who received more than } 1 \text { course of colistin } \\
\text { therapy, } \mathrm{n}(\%)\end{array}$ & $10(23.8 \%)$ & $6(8.2 \%)$ & 0.02 \\
\hline Length of hospital stay (days), median (range) & $46.5(12-246)$ & $32(6-192)$ & 0.04 \\
\hline All-cause mortality at hospital discharge, $\mathrm{n}(\%)$ & $35(47.9 \%)$ & $19(45.2 \%)$ & 0.78 \\
\hline
\end{tabular}

this study was much lower than that of CoR KP isolates. A study from China reported the presence of colistin-resistant Enterobacteriaceae in healthy adults [20]. Subjects with CoR EC or CoR KP colonization at study enrollment in the present study may have had colonized CoR EC or CoR KP in their guts from prior hospitalizations. The transmission of CoR EC or CoR KP from other hospitalized patients with colonization or infections caused by these bacteria and/or from the hospital environment to subjects during hospitalization prior to study enrollment could not be excluded. The use of fluoroquinolones, vancomycin, or aminoglycosides, all of which are non-beta-lactam antibiotics, for the current hospitalization prior to study enrollment may have contributed to the development of CoR EC or CoR KP colonization at study enrollment, because these antibiotics were found to be or tended to be associated with subjects who had CoR EC or CoR KP colonization at study enrollment. Interestingly, the use of beta-lactams during the current hospitalization prior to study enrollment was not found to be significantly associated with CoR EC or CoR KP colonization at study enrollment. Colistin use within 3 months prior to study enrollment may also have contributed to the development of CoR EC or CoR KP colonization at study enrollment, although 
potential statistical significance could not be achieved due to the small size of our study population. CoR EC or CoR KP colonization could have developed in some subjects in the present study after exposure to colistin for only 3 days, which would be consistent with our previous observations in swine that showed colistin-resistant Enterobacteriaceae colonization in the guts of piglets that received colistin for only several days. Association between the emergence of CoR EC or CoR KP and the use of broad-spectrum antibiotics and colistin was previously reported [3, 11, 17, 21]. Colistin-resistant bacteria in patients with no history of colistin use was also reported [22-24].

In patients with no CoR EC or CoR KP colonization at study enrollment that developed CoR EC or CoR KP colonization after study enrollment, it appears evident that colistin use was the main contributing factor, because colistin use within 3 months prior to study enrollment and a larger total dose of colistin, longer duration of colistin, and multiple courses of colistin were all significantly associated with subjects who developed CoR EC or CoR KP colonization after study enrollment. In addition to the use of colistin, transmission of CoR EC or CoR KP from other hospitalized patients with colonization or infection with these colistin-resistant bacteria and/or from the hospital environment during hospitalization could also be potential causes of CoR EC or CoR KP colonization. Since the present study did not have a concurrent cohort of hospitalized patients who did not receive colistin, the effect and magnitude of these other potential causes of CoR EC or CoR KP colonization (in addition to colistin exposure) could not be quantified. Our previous study in CRE colonization and infections in hospitalized patients at our center revealed that approximately $10 \%$ of patients had colistin-resistant Enterobacteriaceae during their hospitalization (V. Thamlikitkul. unpublished data). Therefore, colistin use should still be considered a major contributing driver of CoR EC or CoR KP colonization in subjects who receive colistin. Two previous studies reported emergence of colistin resistance in $K$. pneumoniae after the use of colistin for digestive tract decontamination $[25,26]$. Selective pressure of colistin use in these patients is likely to be one of the contributing factors for this emergence of colistin resistance.

CoR EC or CoR KP was not isolated from $34.8 \%$ of subjects with CoR EC or KP colonization at the end of the study period. The median and range of the duration of CoR EC or CoR KP colonization in these subjects were 18.5 days and 2-68 days, respectively. It should be kept in mind that collection of surveillance culture samples was not performed on a daily basis, but they were collected at enrollment, on days 3 and 7 after the start of colistin treatment, and once a week thereafter. As such, the reported duration from the first sample collection until the disappearance of CoR EC or CoR KP does not represent the real duration of colonization. The duration of colonization could be shorter than the reported duration since CoR EC or CoR KP could disappear between the sample collection times. These observations indicate that colistin resistance could be disappeared, which is similar to colistin resistance findings in Enterobacteriaceae studies in piglets that rapidly developed resistance when colistin was given, but the resistance was usually disappeared once colistin was discontinued (V. Thamlikitkul. unpublished data). Regarding subjects with persistent CoR EC or CoR KP colonization at the end of the study period, colonization duration data was not available since these patients were not followed beyond the end of the study period. The duration of colistin exposure in patients with persistent CoR EC or CoR KP colonization at the end of the study seemed to be longer than in those with no observable CoR EC or CoR KP colonization at the end of the study.

The mechanisms of colistin resistance in Enterobacteriaceae should be different from those in non-fermentative Gram-negative bacteria, especially $A$. baumannii and $P$. aeruginosa. A. baumannii and $P$. aeruginosa are far less likely to develop colistin resistance during exposure to colistin, because the prevalence of colistin-resistant $A$. baumannii-caused and $P$. aeruginosa-caused infections in hospitalized patients at Siriraj Hospital has been less than $5 \%$ even though colistin has been widely used for treatment of carbapenem-resistant $A$. baumannii and $P$. aeruginosa infections at Siriraj Hospital for longer than 10 years [27]. In contrast, brief exposure by $E$. coli and $K$. pneumoniae to colistin in hospitalized patients was associated with colistin resistance in E. coli and K. pneumoniae, as was observed in the present study and in the previous study that investigated colistin use in swine.

Although the number of CoR KP isolates was far larger than the number of CoR EC isolates in our study population, the prevalence of the mcr-1 gene in CoR KP isolates (7.7\%) was much lower than in CoR EC isolates (57.7\%). This observation was similar to observations from our previous study that the mcr-1 gene was detected in only $1.4 \%$ of 280 suspected CoR KP isolates, whereas it was detected in $29.7 \%$ of 37 suspected CoR EC isolates [28]. Again, similar to data from our previous study, the MICs of colistin in CoR EC isolates with the mcr-1 gene were lower than the colistin MICs in CoR KP isolates with the mcr-1 gene [28]. Since the mcr-1 gene was detected in only some isolates of CoR EC and in only a small portion of CoR KP isolates, CoR EC and CoR KP without the mcr-1 gene isolated from subjects in our study could be resistant to colistin via other types of plasmid-mediated mor genes (including mcr-2, mcr-3, mcr-4, mcr-5), or other resistance mechanisms, or combined mechanisms of resistance [29-33]. We are currently investigating the mechanisms of colistin resistance among CoR EC and KP isolates without the mcr-1 gene. 
CoR EC or CoR KP colonization in hospitalized patients could pose multiple threats to their own wellbeing and the wellbeing of others. Although this study found only 3 subjects who had CoR KP colonization and infection, hospitalized patients with CoR EC or CoR KP colonization should be considered to be at risk of developing infections due to CoR EC or CoR KP at a later time point. This later risk of infection was observed in neutropenic patients, in other immunocompromized patients or critically-ill patients who developed subsequent multidrug-resistant infections after colonization with the same multidrug-resistant bacteria, and surveillance culture collected from such patients to determine the presence of multidrug-resistant colonization was suggested because intestinal colonization with multidrug-resistant bacteria was found to be associated with subsequent infections caused by the same colonized multidrug-resistant bacteria [34-37]. Hospitalized patients with CoR EC or CoR KP colonization could transmit these colistin-resistant bacteria to other hospitalized patients, healthcare personnel, their relatives, and into the hospital environment. Patients with an antibiotic-resistant plasmid-mediated gene, such as mcr-1, in colonized bacteria could transfer this genetic mobile element to other bacteria residing in their body, other patients, or the hospital environment, which could result in further emergence and spread of colistin-resistant pathogens. Patients with persistent colonization of CoR EC or CoR KP could transmit these bacteria to others living in their home and into the environment in their community when they return home from the hospital, and these colistin-resistant bacteria may cause community-acquired infection in the future. The length of hospital stay in subjects with CoR EC or CoR KP colonization was significantly longer than the length of stay among subjects without CoR EC or CoR KP colonization; however, the all-cause mortality rate at hospital discharge was similar between subjects with and without CoR EC or CoR KP colonization.

The outcomes of infections caused by CoR EC or CoR $\mathrm{KP}$ are not always unfavorable or fatal. Many isolates of CoR EC and some isolates of CoR KP recovered from subjects in this study were still susceptible to carbapenems. Although all 5 subjects infected with CoR KP had infections caused by carbapenem-resistant isolates, all of them received combination of colistin and fosfomycin or aminoglycosides and they were alive at hospital discharge. Infection caused by CoR EC was not observed in this study. This may be mostly and simply due to the fact that there was only a small number of subjects who had colonization with CoR EC compared to a much more number of subjects with CoR KP colonization.

Polymyxins will continue to be a mainstay antimicrobial group for therapy of carbapenem-resistant $A$. baumannii and $P$. aeruginosa infections, both of which are very prevalent in hospital-acquired infections in many countries, including Thailand. Polymyxins will also be the treatment of choice for CRE infection, which has been an emerging hospital-acquired infection in Thailand over the past 5 years, since more effective, more efficient, and safer agents for treatment of these carbapenem-resistant Gram-negative infections are not yet available. The responsible physician should prescribe colistin for therapy of documented carbapenem-resistant Gram-negative infections that are susceptible to colistin at the shortest duration of treatment. Colistin should not be used in patients with carbapenem-resistant Gram-negative colonization, and it should be used as empirical antimicrobial therapy as rarely as possible. These strategies for the use of colistin may minimize collateral damage caused by the use of colistin regarding the development and transmission of $\mathrm{CoR}$ EC or CoR KP in both healthcare and community settings. However, even appropriate use of colistin is still a key driver of the inevitable emergence of CoR EC or CoR KP. The high prevalence of CoR EC or CoR KP colonization observed in this study is worrisome, and it may predict an increase in CoR EC or CoR KP infections in the future. The heavy use of colistin in hospitalized patients may result in infections caused by CoR EC or CoR KP becoming endemic in healthcare facilities in the near future.

\section{Conclusions}

Prevalence of CoR EC or CoR KP colonization in hospitalized patients receiving colistin was high and it was associated with the use of colistin. Therefore, patients who receive colistin are at risk of developing CoR EC or CoR $\mathrm{KP}$ colonization and infection.

\section{Acknowledgements \\ The authors gratefully acknowledge Chulaluk Komoltri for assistance with statistical analysis. \\ Funding \\ This study was funded by grants from the Health Systems Research Institute, Thailand and the Faculty of Medicine Siriraj Hospital, Mahidol University. \\ Availability of data and materials \\ The datasets used and/or analysed during the current study are available from the corresponding author on reasonable request. \\ Authors' contributions \\ WW and $\mathrm{VT}$ contributed to conception and design of the article. NP, NM CS and ST were responsible for microbiological tests. WW, NP, NM CS, ST and VT collected and analysed data. WW and VT wrote the first version of the manuscript. All authors critically revised the manuscript, and read and approved the final manuscript.}

\section{Ethics approval and consent to participate}

The study protocol was approved by the Siriraj Institutional Review Board, and written informed consent was obtained from all subjects.

Consent for publication

Not applicable.

Competing interests

The authors declare that they have no competing interests 


\section{Publisher's Note}

Springer Nature remains neutral with regard to jurisdictional claims in published maps and institutional affiliations.

\section{Author details}

${ }^{1}$ Division of Infectious Diseases and Tropical Medicine, Department of Medicine, Faculty of Medicine Siriraj Hospital, Mahidol University, 2 Wanglang Road, Bangkoknoi, Bangkok 10700, Thailand. ${ }^{2}$ Department of Clinical Microbiology and Applied Technology, Faculty of Medical Technology, Mahidol University, Bangkok, Thailand.

Received: 6 June 2018 Accepted: 4 July 2018

Published online: 17 July 2018

\section{References}

1. Biswas S, Brunel JM, Dubus JC, Reynaud-Gaubert M, Rolain JM. Colistin: an update on the antibiotic of the 21st century. Expert Rev Anti-Infect Ther. 2012;10:917-34.

2. Catry B, Cavaleri M, Baptiste K, Grave K, Grein K, Holm A, Jukes H, Liebana E, et al. Use of colistin-containing products within the European Union and European economic area (EU/EEA): development of resistance in animals and possible impact on human and animal health. Int J Antimicrob Agents. 2015;46:297-306.

3. Kontopidou F, Plachouras D, Papadomichelakis E, Koukos G, Galani I Poulakou G, et al. Colonization and infection by colistin-resistant gramnegative bacteria in a cohort of critically ill patients. Clin Microbiol Infect. 2011;17:E9-e11.

4. Rhouma M, Beaudry F, Theriault W, Letellier A. Colistin in pig production: chemistry, mechanism of antibacterial action, microbial resistance emergence, and one health perspectives. Front Microbiol. 2016;7:1789.

5. Antoniadou A, Kontopidou F, Poulakou G, Koratzanis E, Galani I, Papadomichelakis E, et al. Colistin-resistant isolates of Klebsiella pneumoniae emerging in intensive care unit patients: first report of a multiclonal cluster. J Antimicrob Chemother. 2007;59:786-90.

6. Olaitan AO, Morand S, Rolain JM. Mechanisms of polymyxin resistance: acquired and intrinsic resistance in bacteria. Front Microbiol. 2014;5:643.

7. Liu YY, Wang Y, Walsh TR, Yi LX, Zhang R, Spencer J, et al. Emergence of plasmid-mediated colistin resistance mechanism MCR-1 in animals and human beings in China: a microbiological and molecular biological study. Lancet Infect Dis. 2016;16:161-8.

8. Wang $R$, van Dorp L, Shaw LP, Bradley P, Wang Q, Wang X, Jin L, Zhang $Q$, Liu Y, Rieux A, Dorai-Schneiders T, Weinert LA, lqbal Z, Didelot X, Wang H, Balloux $F$. The global distribution and spread of the mobilized colistin resistance gene mcr-1. Nat Commun. 2018;9:1179.

9. Qureshi ZA, Hittle LE, O'Hara JA, Rivera JI, Syed A, Shields RK, et al. Colistinresistant Acinetobacter baumannii: beyond carbapenem resistance. Clin Infect Dis. 2015;60:1295-303.

10. Inchai J, Liwsrisakun C, Theerakittikul T, Chaiwarith R, Khositsakulchai W, Pothirat $C$. Risk factors of multidrug-resistant, extensively drug-resistant and pandrug-resistant Acinetobacter baumannii ventilator-associated pneumonia in a medical intensive care unit of University Hospital in Thailand. J Infect Chemother. 2015;21:570-4.

11. Matthaiou DK, Michalopoulos A, Rafailidis PI, Karageorgopoulos DE, Papaioannou V, Ntani G, et al. Risk factors associated with the isolation of colistin-resistant gram-negative bacteria: a matched case-control study. Crit Care Med. 2008;36:807-11.

12. Durante-Mangoni E, Del Franco M, Andini R, Bernardo M, Giannouli M, Zarrilli R. Emergence of colistin resistance without loss of fitness and virulence after prolonged colistin administration in a patient with extensively drug-resistant Acinetobacter baumannii. Diagn Microbiol Infect Dis. 2015:82:222-6.

13. Kim Y, Bae IK, Jeong SH, Yong D, Lee K. In vivo selection of pan-drug resistant Acinetobacter baumannii during antibiotic treatment. Yonsei Med J. 2015:56:928-34

14. Farmer JJ 3rd, Davis BR, Hickman-Brenner FW, McWhorter A, Huntley-Carter GP, Asbury MA, et al. Biochemical identification of new species and biogroups of Enterobacteriaceae isolated from clinical specimens. J Clin Microbiol. 1985:21:46-76.

15. Juhasz E, Ivan M, Pinter E, Pongracz J, Kristof K. Colistin resistance among blood culture isolates at a tertiary care Centre in Hungary. J Glob Antimicrob Resist. 2017;11:167-70.
16. Prim N, Turbau M, Rivera A, Rodriguez-Navarro J, Coll P, Mirelis B. Prevalence of colistin resistance in clinical isolates of Enterobacteriaceae: a four-year cross-sectional study. J Inf Secur. 2017;75:493-8.

17. Papadimitriou-Olivgeris M, Christofidou M, Fligou F, Bartzavali C, Vrettos T, Filos KS, et al. The role of colonization pressure in the dissemination of colistin or tigecycline resistant KPC-producing Klebsiella pneumoniae in critically ill patients. Infection. 2014;42:883-90.

18. Boonyasiri A, Tangkoskul T, Seenama C, Saiyarin J, Tiengrim S, Thamlikitkul V. Prevalence of antibiotic resistant bacteria in healthy adults, foods, food animals, and the environment in selected areas in Thailand. Pathog Glob Health. 2014;108:235-45.

19. Khamsarn S, Nampoonsak Y, Busamaro S, Tangkoskul T, Seenama C, Rattanaumpawan P, Boonyasiri A, Thamlikitkul V. Epidemiology of antibiotic use and antimicrobial resistance in selected communities in Thailand. J Med Assoc Thail. 2016;99:270-5.

20. Wang $Y$, Tian GB, Zhang $R$, Shen $Y$, Tyrrell JM, Huang $X$, et al. Prevalence, risk factors, outcomes, and molecular epidemiology of mcr-1-positive Enterobacteriaceae in patients and healthy adults from China: an epidemiological and clinical study. Lancet Infect Dis. 2017;17:390-9.

21. Giacobbe DR, Del Bono V, Trecarichi EM, De Rosa FG, Giannella M, Bassetti $M$, et al. Risk factors for bloodstream infections due to colistin-resistant KPCproducing Klebsiella pneumoniae: results from a multicenter case-controlcontrol study. Clin Microbiol Infect. 2015;21:1106.e1-8.

22. Olaitan AO, Morand S, Rolain JM. Emergence of colistin-resistant bacteria in humans without colistin usage: a new worry and cause for vigilance. Int J Antimicrob Agents. 2016;47:1-3.

23. Chen S, Hu F, Zhang X, Xu X, Liu Y, Zhu D, et al. Independent emergence of colistin-resistant Enterobacteriaceae clinical isolates without colistin treatment. J Clin Microbiol. 2011;49:4022-3.

24. Nordmann P, Lienhard R, Kieffer N, Clerc O, Poirel L. Plasmid-mediated colistin-resistant Escherichia coli in bacteremia in Switzerland. Clin Infect Dis. 2016:62:1322-3.

25. Lubbert C, Faucheux S, Becker-Rux D, Laudi S, Durrbeck A, Busch T, et al. Rapid emergence of secondary resistance to gentamicin and colistin following selective digestive decontamination in patients with KPC-2producing Klebsiella pneumoniae: a single-Centre experience. Int J Antimicrob Agents. 2013;42:565-70.

26. Halaby T, Al Naiemi N, Kluytmans J, van der Palen J, Vandenbroucke-Grauls CM. Emergence of colistin resistance in Enterobacteriaceae after the introduction of selective digestive tract decontamination in an intensive care unit. Antimicrob Agents Chemother. 2013;57:3224-9.

27. Thamlikitkul V, Popum S. Monitoring of effectiveness and safety of Colistin for therapy in resistant gram-negative bacterial infections in hospitalized patients at Siriraj hospital. J Med Assoc Thail. 2016;99:301-7.

28. Eiamphungporn W, Yainoy S, Jumderm C, Tan-arsuwongkul R, Tiengrim S, Thamlikitkul V. Prevalence of colistin resistance gene mcr-1 in colistinresistant Escherichia coli and Klebsiella pneumoniae isolated from humans in Thailand. J Glob Antimicrob Resist. 2018; (In press)

29. Quan J, Li X, Chen Y, Jiang Y, Zhou Z, Zhang H, et al. Prevalence of mcr-1 in Escherichia coli and Klebsiella pneumoniae recovered from bloodstream infections in China: a multicentre longitudinal study. Lancet Infect Dis. 2017; 17:400-10.

30. Xavier BB, Lammens C, Ruhal R, Kumar-Singh S, Butaye P, Goossens H, et al. Identification of a novel plasmid-mediated colistin-resistance gene, mcr-2, in Escherichia coli, Belgium, June 2016. Euro Surveill. 2016;21:27.

31. Yin W, Li H, Shen Y, Liu Z, Wang S, Shen Z, et al. Novel plasmid-mediated colistin resistance gene mcr-3 in Escherichia coli. MBio. 2017;8(4)

32. Carattoli A, Villa L, Feudi C, Curcio L, Orsini S, Luppi A, et al. Novel plasmidmediated colistin resistance mcr-4 gene in Salmonella and Escherichia coli, Italy 2013, Spain and Belgium, 2015 to 2016. Euro Surveill. 2017;22(31)

33. Borowiak M, Fischer J, Hammerl JA, Hendriksen RS, Szabo I, Malorny B. Identification of a novel transposon-associated phosphoethanolamine transferase gene, mcr-5, conferring colistin resistance in d-tartrate fermenting Salmonella enterica subsp. enterica serovar Paratyphi B. J Antimicrob Chemother. 2017;72:3317-24.

34. Schimpff SC, Young VM, Greene WH, Vermeulen GD, Moody MR, Wiernik $\mathrm{PH}$. Origin of infection in acute nonlymphocytic leukemia. Significance of hospital acquisition of potential pathogens. Ann Intern Med. 1972;77:707-14.

35. Wingard JR, Dick J, Charache P, Saral R. Antibiotic-resistant bacteria in surveillance stool cultures of patients with prolonged neutropenia. Antimicrob Agents Chemother. 1986;30:435-9. 
36. Pena C, Pujol M, Ardanuy C, Ricart A, Pallares R, Linares J, et al.

Epidemiology and successful control of a large outbreak due to Klebsiella pneumoniae producing extended-spectrum beta-lactamases. Antimicrob Agents Chemother. 1998:42:53-8.

37. Tancrede $\mathrm{CH}$, Andremont $\mathrm{AO}$. Bacterial translocation and gram-negative bacteremia in patients with hematological malignancies. J Infect Dis. 1985 152:99-103.

Ready to submit your research? Choose BMC and benefit from:

- fast, convenient online submission

- thorough peer review by experienced researchers in your field

- rapid publication on acceptance

- support for research data, including large and complex data types

- gold Open Access which fosters wider collaboration and increased citations

- maximum visibility for your research: over $100 \mathrm{M}$ website views per year

At BMC, research is always in progress.

Learn more biomedcentral.com/submissions 